\title{
Spectroscopic properties of Holmium-Aluminum-Germanium co-doped silica fiber
}

\author{
Yuri O. Barmenkov" ${ }^{\mathrm{a}}$, Alexander V. Kir'yanov ${ }^{\mathrm{a}}$, and Miguel V. Andrés ${ }^{\mathrm{b}}$ \\ ${ }^{a}$ Centro de Investigaciones en Óptica, A.C., Loma del Bosque 115, Col. Lomas del Campestre, 37150, León, \\ Guanajuato, México; ${ }^{\text {b}}$ Departamento de Física Aplicada, Instituto de Ciencia de Materiales, Universidad de \\ Valencia, 46100 Valencia, Spain
}

\begin{abstract}
We report the basic spectroscopic properties of a home-made holmium-aluminum-germanium co-doped silica fiber, designed for laser applications. We present the ground-state and excitedstate absorption spectra of the fiber along with the up-conversion emission spectrum under laser action, which served for making an energy levels' diagram of $\mathrm{Ho}^{3+}$ ion. We discuss the ways to assess the kinetics of $\mathrm{Ho}^{3+} 2$ - $\mu \mathrm{m}$ fluorescence, focusing on measuring fluorescence lifetime from ${ }^{5} \mathrm{I}_{7}$ level and nonradiative decay time from ${ }^{5} \mathrm{I}_{6}$ level. The simple analytical formulae, applicable for fitting of the fluorescence decay curve, are derived, which can be useful for precise modeling of holmium-doped fiber lasers.
\end{abstract}

Keywords: holmium-doped silica fiber, ground-state absorption, excited state absorption, upconversion emission, fluorescence lifetime.

\section{Introduction}

Laser sources operating in the $2 \mu \mathrm{m}$ spectral band are of increasing interest due to numerous industrial, medical, scientific, military, and other applications. For instance, such sources are reported to be successful for processing plastic materials, transparent in visible [1,2], and semiconductor wavers [3], controlling climate behavior [4], remote sensing [5], etc. Significant value of absorption at $\sim 2 \mu \mathrm{m}$ in water and so in biological tissues provides little penetration depth (of the order of $0.1 \mathrm{~mm}$ ) causes remarkable usefulness of 2- $\mu \mathrm{m}$ lasers for medical applications, including laser surgery [6].

Fiber lasers (FLs) demonstrate some essential features, lacking in other types of lasers. These are associated with superior brightness, resulting from the fiber waveguide nature, high one-pass gain, applicability of double-clad fiber geometry for effective high-power pumping, efficient power budget and thermal control, and easy incorporation to a compact package. All this makes FLs indispensable.

Thulium- and holmium-doped silica FLs operate in the 2- $\mu \mathrm{m}$ optical band, just below the multi-phonon (infrared) absorption edge, inherent to silica glass $(\sim 2.4 \mu \mathrm{m})$. Although the first experimental implementations of such FLs were reported more than 30 years ago [7-10], currently, thulium-doped FLs (TDFLs) are the most developed and thus most frequently used 2$\mu \mathrm{m}$ FL sources. In the meantime, there are some important benefits that holmium-doped FLs (HDFLs) have over TDFLs: for instance, HDFLs are capable to oscillate beyond $2.1 \mu \mathrm{m}$ [11], the range of wavelengths, non-assessable in TDFLs. Since HDFLs are not so precisely developed as 
TDFLs, some clue optical parameters of HDF to be referred to at making laser optimization using numerical analyses are either unknown or their values largely scatter in references.

HDFLs are usually pumped either employing TDFLs (at wavelengths slightly below $2 \mu \mathrm{m}$, i.e. into $\mathrm{Ho}^{3+}$ laser level ${ }^{5} \mathrm{I}_{7}$ [12-14]), or ytterbium-doped FLs (YDFLs) (at 1.12 ..1.15 $\mu$ m, i.e. into its higher-laying level ${ }^{5} \mathrm{I}_{6}$ [14-19]). The first pumping option is more efficient and commonly used due to its relative simplicity; the second one is barely developed due to technical problems, associated with the obvious difficulties to bring YDFLs in operation at wavelengths beyond 1.1 $\mu \mathrm{m}[19]$.

In this work, we report an experimental study of the basic optical properties of a home-made HDF. First, we demonstrate the HDF's broadband ground-state absorption (GSA) spectrum along with the excited-state absorption (ESA) and spontaneous emission (SE) spectra under pumping at $\sim 1.12 \ldots 1.135 \mu \mathrm{m}$ as a background for analyzing the transitions of $\mathrm{Ho}^{3+}$ ions, involved under laser action. Then, we propose and discuss an appropriate theory that addresses decaying of $\mathrm{Ho}^{3+}$ ion from the laser level, ${ }^{5} \mathrm{I}_{7}$, to the ground one, ${ }^{5} \mathrm{I}_{8}$, in the circumstances of simultaneous populating of the former from the level ${ }^{5} \mathrm{I}_{6}$ under $1.12 \ldots 1.135-\mu \mathrm{m}$ pumping and subsequent reabsorption of spontaneous emission (SE), implying that the condition of optically short HDF (or smallness of optical depth $(O D)$ ) is not satisfied through its length. As a result, we show that the knowledge of $O D$-value and availability of a complete SE decay curve permit one to obtain the fluorescence lifetime from ${ }^{5} \mathrm{I}_{7}$ (laser) level and, also, the rate of multi-phonon relaxation time from ${ }^{5} \mathrm{I}_{6}$ (pump) level. Also, we demonstrate that, if $O D$-value is equal to a few tenths of unit, the lifetime value measured using the exponential fitting is by tens of percent less than its real value; the longer the fiber under test, the greater the error that arises at lifetime measurements. We also derive an analytical formula for the SE decay process in the mentioned circumstances, which can be applied for fitting a detected signal of SE light, collected from a relatively long HDF ( $O D$ is not small), posterior to fast turning off the pump light. This formula is applicable for measuring lifetime of other rare-earth doped fibers with relatively large $O D$.

\section{HDF fabrication and its waveguide properties}

The preform of the home-made HDF was fabricated using the surface plasma chemical vapor deposition (SPCVD) method [20] and the final fiber was drawn using a standard fiber draw tower. The HDF preform was also co-doped with $\mathrm{Al}$ and $\mathrm{Ge}$ for managing numerical aperture $\mathrm{NA}$ and $(\mathrm{Al})$ to reduce $\mathrm{Ho}^{3+}$ clustering. Concentrations of $\mathrm{Ho}, \mathrm{Al}$, and $\mathrm{Ge}$ in the preform, found using an electron-probe microanalyzer, were $\sim 0.03 \mathrm{~mol} . \%, \sim 0.75 \mathrm{~mol} . \%$, and $\sim 1.2 \mathrm{~mol} . \%$, respectively; thus, Al/Ho ratio was $\sim 25$. The HDF's small-signal absorption peak at $1.95 \mu \mathrm{m}$ was $A b s=15 \mathrm{~dB} / \mathrm{m}$ (see Fig. 1 below) that corresponds to the absorption constant $\alpha_{0}=3.45 \mathrm{~m}^{-1}$. The fabricating process details are reported in refs. [17-18].

The HDF's core diameter was $10.5 \mu \mathrm{m}$, cut-off wavelength was $1.95 \mu \mathrm{m}$, and $N A$ was 0.14 . This set of waveguide parameters corresponds to the large overlap factor of the fundamental mode $\mathrm{LP}_{01}$ with the fiber core $(\Gamma=0.78)$ for lasing beyond $2 \mu \mathrm{m}$ and, accordingly, a high fiber gain. Considering $\mathrm{Ho}^{3+}$ absorption cross-section at $1.95 \mu \mathrm{m} \sigma_{a b s}=3 \times 10^{-25} \mathrm{~m}^{2}[21,22]$, $\mathrm{Ho}^{3+}$ effective core concentration was calculated to be $N_{0}=\alpha_{0} /\left(\Gamma \sigma_{a b s}\right)=1.47 \times 10^{25} \mathrm{~m}^{-3}$. Note that the value of $\sigma_{a b s}$ is still a subject of further assessment as it dramatically disperses in the available literature, see, for instance, refs. [21,23-26].

\section{HDF absorption and emission spectra}


The HDF's GSA spectra were measured using a white light source (Yokogawa Q4305) with a FC/PC mating sleeve as an output fiber adapter and three optical spectrum analyzers (OSAs): Yokogawa AQ6370B, Yokogawa AQ6375B, and ANDO AQ6315A for the optical bands 600$1700 \mathrm{~nm}, 1200-2400 \mathrm{~nm}$, and 400-600 nm, respectively. The absorption spectra were measured using HDFs of different lengths (and different $O D s$ ); the final spectrum obtained by matching the spectra, obtained with the three OSAs, is shown in Fig. 1(a) by the blue curve (for the range 400 $\mathrm{nm}$ to $1300 \mathrm{~nm}$ ) and by the magenta curve (for the range $1300 \mathrm{~nm}$ to $2400 \mathrm{~nm}$ ); note that both axes in this plot use logarithmic scales.

The energy level system of $\mathrm{Ho}^{3+}$ ion is shown in Fig. 2. The levels are drawn in accordance to the photon energy of the corresponding absorption GSA peaks. The levels marked as 1, 2, and 3 are the ground, laser, and pump levels; we use here the term "pump" for the third $\mathrm{Ho}^{3+}$ level as in our experiments the pump sources used were within the range 1.12 to $1.135 \mu \mathrm{m}$, for which photon energy corresponds to the transition ${ }^{5} \mathrm{I}_{8} \rightarrow{ }^{5} \mathrm{I}_{6}$. The levels with the energies superior of $2.4 \times 10^{4} \mathrm{~cm}^{-1}$, or wavelengths below $400 \mathrm{~nm}$, are not shown (due to incapability of measurements using the available OSAs).
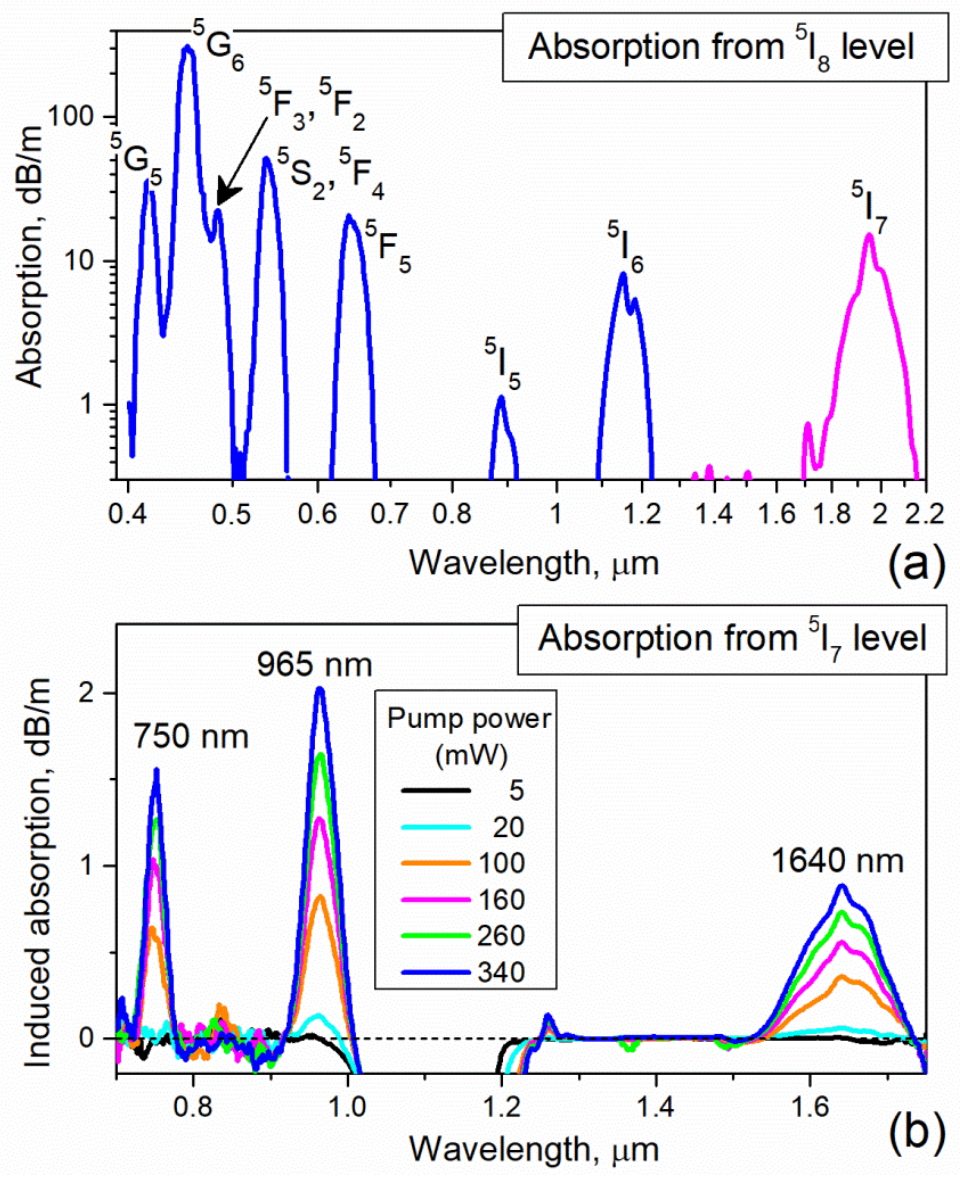

Figure 1. (a) GSA spectrum of HDF under study; its parts measured using OSAs AQ6370B and AQ6375B are highlighted by blue and magenta curves, respectively. (b) ESA spectra under pumping at $1.12 \mu \mathrm{m}$ with incident powers indicated in inset (the artifact of negative peak at $1.12 \mu \mathrm{m}$ is associated with the pump line). At measuring both the plots, OSA resolution was set to $2 \mathrm{~nm}$. 


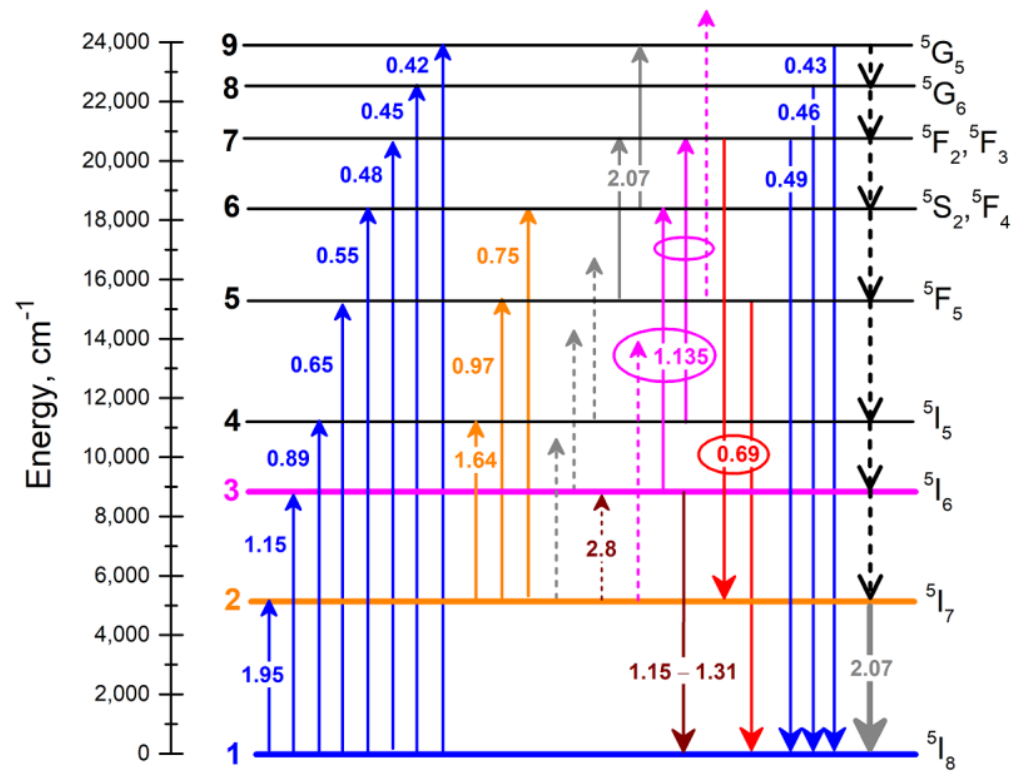

Figure 2. Energy diagram of the $\mathrm{Ho}^{3+}$ system; the energy levels are labeled on the right and, also, are numbered in ascending order from bottom to top on the left. The inter-level transitions, highlighted by the arrows of different colors, are specified and discussed in the text.

The blue (upward) arrows stand for the transitions from the ground state, with the related wavelengths (in $\mu \mathrm{m}$ ) given near each arrow; these wavelengths were found from the optical spectrum shown in Fig. 1(a).

The orange arrows show the transitions from the $\mathrm{Ho}^{3+}$ excited (in fact, laser) state ${ }^{5} \mathrm{I}_{7}$. The ESA transitions are shown in Fig. 1(b). The spectrum shown here was calculated as the additional white-light attenuation arising in the HDF in the presence of pump light at $1.12 \mu \mathrm{m}$ (to get this spectrum, we used a wavelength division multiplexer (WDM) for separating the pump and white light paths). As seen, the ESA peaks' magnitudes depend on pump power: the stronger pump, the greater the magnitudes are. This is explained by the fact that population of the laser level $\left({ }^{5} \mathrm{I}_{7}\right)$ increases with increasing pump power, with the result being growth of density of the transitions sourced by this level in the conditions of the same spectral density of probe white light. Note that the ESA spectrum is demonstrated in the wavelength range limited from the right by $1750 \mathrm{~nm}$ because of amplified white light (test) signal observed beyond this wavelength as negative absorption that does not permit one to detect possible ASE lines in this range.

The gray (solid and dash) arrows stand for the transitions at "common" HDFL wavelengths $(2.05$ to $2.07 \mu \mathrm{m})$. Note that the transitions shown by the dash arrows are less probable at the laser photon energy $\left(\sim 4830 \mathrm{~cm}^{-3}\right)$ unequal to the energy gaps between the correspondent $\mathrm{Ho}^{3+}$ energy levels whilst the ones shown by the solid arrows are more probable at their matching. Thus, ESA at the laser wavelength is not high neither on the laser nor on the pump wavelength.

The dark-red dot arrow stands for the transition with wavelength of $2.8 \mu \mathrm{m}$, which is not occurring due to the high multi-phonon absorption of silica glass beyond $2.4 \mu \mathrm{m}$.

The magenta arrows stand for the transitions upon pump absorption at $1.13 \mu \mathrm{m}$, with the dash and solid ones showing less and more probable transitions, respectively. It is important that the pump-photon energy matches well with the energy gap between the pump $\left({ }^{5} \mathrm{I}_{6}\right)$ and the sixth levels and, also, between the fourth and seventh levels. Thus, ESA at the pump wavelength 
occurs directly from the pump level and then, with a lower probability, from the next upper level after multi-phonon nonradiative decays.

The red and blue (downward) arrows stand for the SE transition at $0.69 \mu \mathrm{m}$ and those in the blue spectral range (both discussed below) and the black dash ones for the multi-phonon transitions between the excited states of $\mathrm{Ho}^{3+}$.

Eventually, the gray line marked " $2.07 \mu \mathrm{m}$ " stands for the laser transition.

Our laser experiments showed that the HDF under laser action also fluoresces in the visible, viz. in the red and blue spectral ranges; Fig. 3 reveals the effect.

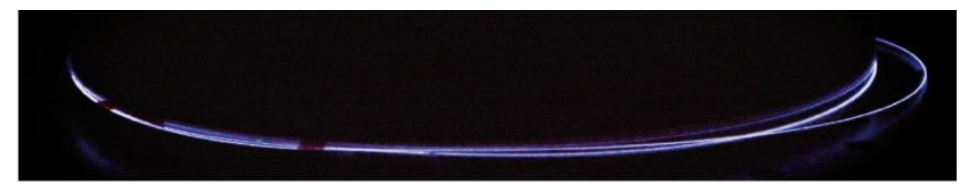

(a)
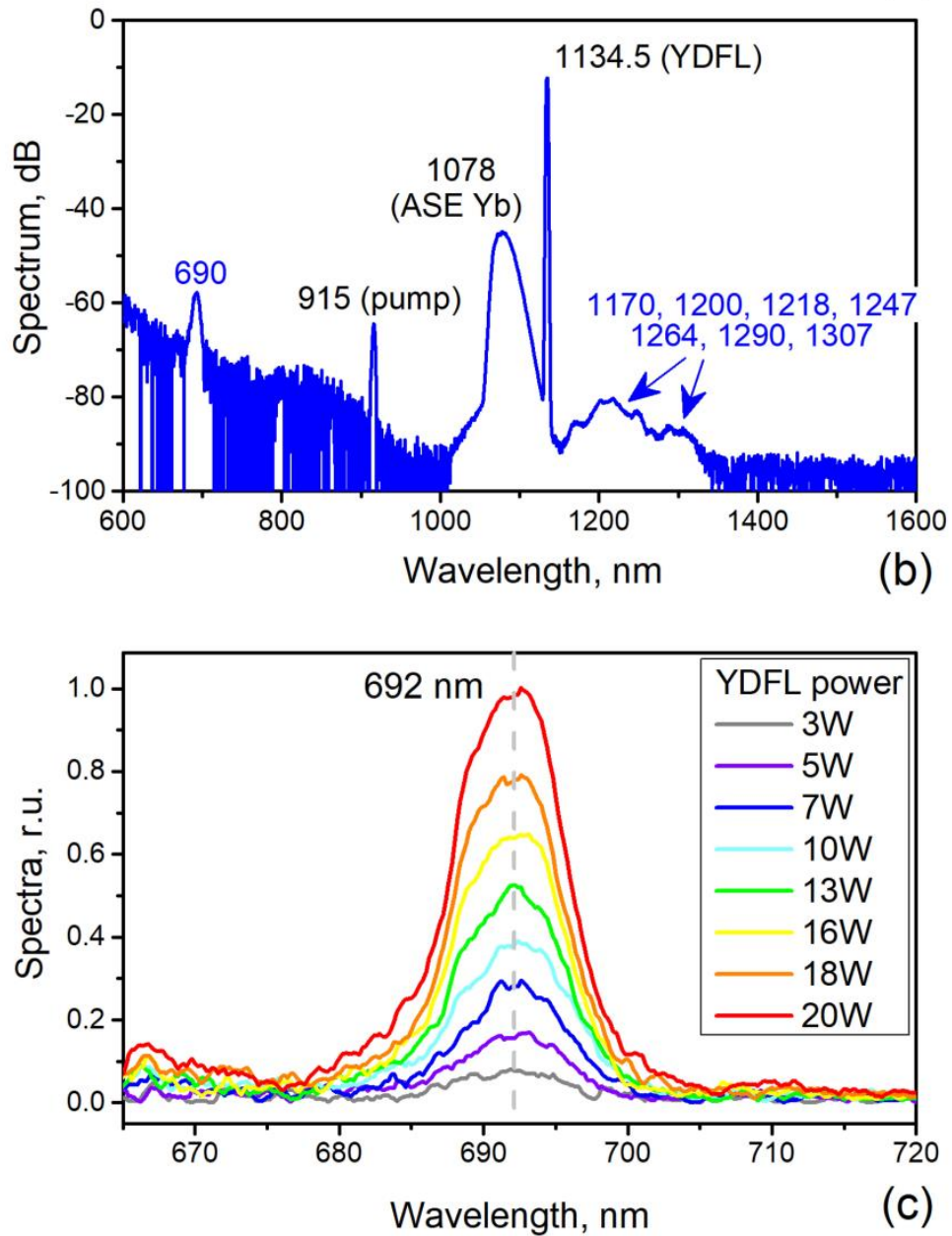

Figure 3. (a) Photo of the fluorescing HDF in the blue to red spectral range at laser action. (b) Broadband spectrum measured at the HDFL output in the range 600-1600 nm. The spectral peaks labeled by the blue numbers are the SE lines and those marked by the black numbers are the residual pump ripples and ASE lines of the pump YDFL. (c) Normalized narrowband HDF emission spectrum, specified for the SE peaking at $692 \mathrm{~nm}$ and measured at different YDFL pump powers @ $1.135 \mu \mathrm{m}$ (see inset). For the plots (b) and (c), OSA resolution was set to $2 \mathrm{~nm}$.

The photo (a) in the figure demonstrates it straightforwardly. 
Furthermore, the plot (b) shows the broadband optical spectrum, measured at the HDFL output below the laser wavelength $(2.07 \mu \mathrm{m})$. As seen, there are three spectral components (with the peak wavelengths marked in black), corresponding to: (i) the residual (unabsorbed) YDFL power at pump $(1.135 \mu \mathrm{m})$ wavelength; (ii) the YDFL amplified spontaneous emission (ASE) line with gravity center at $1078 \mathrm{~nm}$, and (iii) the ripple of the residual power of the 915-nm laser diode, used for pumping the YDFL. The other peaks, marked in Fig. 3 by the blue numbers, correspond to SE at $690 \mathrm{~nm}$ and from 1170 to $1370 \mathrm{~nm}$.

The SE spectra at $690 \mathrm{~nm}$, measured at different pump powers (see the inset), are zoomed in Fig. 3(c). As seen, SE intensity at this wavelength increases with increasing pump power. The energy diagram in Fig. 2 suggests that there are the two relaxation processes responsible for this SE line: one from the seventh energy level to the laser level and another from the fifth energy level to the ground state.

Spectra of the blue luminescence cannot be discussed in detail as these were not detected because of the limitations of the spectral apparatus. Here we can only argue that this luminescence is basically sourced by the seventh $\mathrm{Ho}^{3+}$ level while its contributions sourced by the eighth and ninth levels are minor as the energy gap between the seventh and sixth levels $\left(\sim 2150 \mathrm{~cm}^{-1}\right)$ is larger than that between the adjacent upper levels $\left(\sim 1650 \mathrm{~cm}^{-1}\right.$ and $\left.\sim 1600 \mathrm{~cm}^{-1}\right)$; so, multi-phonon excitation decay of the seventh level is less probable than that of the upper lying levels [27,28] and hence its population is effectively higher.

The luminescence beyond $1600 \mathrm{~nm}$ corresponds to $\mathrm{Ho}^{3+}$ fluorescence due to transition from

the laser level ${ }^{5} \mathrm{I}_{7}$ to the ground level ${ }^{5} \mathrm{I}_{8}$ already studied in a number of works $[17,18,21,22,25,29,30]$ and therefore are not discussed in the present work.

\section{Lifetimes of ${ }^{5} \mathrm{I}_{6}$ and ${ }^{5} \mathrm{I}_{7}$ levels of $\mathrm{Ho}^{3+}$ ions in silica fiber}

As noted above, the lifetimes of $\mathrm{Ho}^{3+}$ "laser" level ${ }^{5} \mathrm{I}_{7}$ and "pump" level ${ }^{5} \mathrm{I}_{6}$ are the important HDF characteristics for modeling HDFL. However, for silica-based HDFs, there are no data on ${ }^{5} \mathrm{I}_{6}$ lifetime in the available literature and the data on ${ }^{5} \mathrm{I}_{7}$ lifetime, while reported many times [13,22,25,29-33], unsatisfactorily scatter, which can be explained by the differences in fabricating methods employed (solution doping or nanoparticle doping), Al to Ho ratio, Ho concentration, etc.

Below, we show that the mentioned discrepancy in the values of $\mathrm{Ho}^{3+}{ }^{5} \mathrm{I}_{7}$ level's fluorescence lifetime may arise because of ignoring $O D$ of a HDF under study. Another important issue tackled below is the depletion of the pump level, ${ }^{5} \mathrm{I}_{6}$, to the laser one, ${ }^{5} \mathrm{I}_{7}$, via decay on phonons, which results in deviation of fluorescence decay from the exponential law.

\subsection{Theoretical background: influence of large optical depth upon fluorescence lifetime measurement}

First, we address the impacts of HDF's $O D$ and depletion of the pump level upon the measurement of the laser level's lifetime. [34]:

$\mathrm{SE}$ power can be found from the equation for a fiber amplifier with time-dependent gain

$$
\left(\frac{n_{m}}{c} \frac{\partial}{\partial t}-\frac{\partial}{\partial z}\right) P(z, t)=\left(g_{0} n_{2}-\alpha_{0} n_{1}\right) P+\eta \frac{\Omega}{4 \pi} \frac{n_{2}}{\tau_{2}}\left(h v N_{0}\right)\left(\pi a^{2}\right)
$$


where $n_{m}$ is the fiber modal index, $c$ is the velocity of light, $z$ is the SE propagation direction, $P$ is SE power, $g_{0}$ is the saturated gain and $\alpha_{0}$ is the low-signal light absorption, $n_{1}=N_{1} / N_{0}$ and $n_{2}=$ $N_{2} / N_{0}$ are the normalized populations of the ground and laser levels, $\Omega$ is the solid angle of acceptance of SE by the fiber core $(\Omega / 4 \pi \approx 0.024), \tau_{2}$ is the decay time from the laser level, $h v$ is the SE photon energy (accordingly, $h m_{2} N_{0}$ is the fiber charge density in $\mathrm{J} / \mathrm{m}^{3}$ ), $a$ is the fiber core radius, and $\eta(0<\eta<1)$ is the probability (quantum efficiency) of the radiative relaxation of $\mathrm{Ho}^{3+}$ ion, whenever the relaxation pathways are purely radiative or not (quenched on phonons) $[13,28,33]$. As it follows from ref. [31], the quantum efficiency of the photon transition for HDF is $\eta \approx 7 \ldots 10 \%$.

In Eq. (1), the term $\left(g_{0} n_{2}-\alpha_{0} n_{1}\right)$ is the current gain/absorption and $\left(n_{2} N_{0} / \tau_{2}\right)$ is the number of SE photons, emitted by the unit fiber core volume in all directions per the unit time interval. Since the light propagation time along the fiber is much less than the changes in its gain and absorption, the time derivative can be ignored, which allows one to rewrite Eq. (1) as:

$$
\frac{d P}{d z}=\left[\left(\alpha_{0}+g_{0}\right) n_{2}-\alpha_{0}\right] P+K_{s e} n_{2}
$$

where $n_{1}$ was substituted with $\left(1-n_{2}\right) . K_{s e}=\eta(\Omega / 4 \pi)\left(h v N_{0}\right)\left(\pi a^{2}\right) / \tau_{2}$ is the fraction of SE photons, captured by the fiber's core section $d z$ (implying all $\mathrm{Ho}^{3+}$ ions are excited to the laser level). The solution of Eq. (2) can be obtained after integrating by parts:

$$
P_{\text {out }}(t)=K_{\text {se }} \exp \left(-t / \tau_{2}\right) \frac{\exp \left[\left(2 n_{20} \exp \left(-t / \tau_{2}\right)-1\right) O D\right]-1}{2 n_{20} \exp \left(-t / \tau_{2}\right)-1}
$$

Here, $P_{\text {out }}(t)$ is the time-dependent fluorescence power at the detector input at $t>0$. Note that at deriving formula (3), we considered that $g_{0}=\alpha_{0}, O D=\alpha_{0} z$, and $n_{20}=n_{2}(t=0)$ is the ${ }^{5} \mathrm{I}_{7}$ level's population just before pump switching off. We use below for $P_{\text {out }}(t)$ the name complex decay function (CDF); it will be used for fitting.

If $O D$ is very small, then formula (3) takes the form of single exponential decay with the initial value that is proportional to $O D$ :

$$
P_{\text {out }}(t)=K_{\text {se }} O D \exp \left(-t / \tau_{2}\right)
$$

Given that thermal noise of photodetectors at $2-\mu \mathrm{m}$ is much greater than that within the telecommunication band and that HDF SE is reduced due to multi-phonon relaxation of $\mathrm{Ho}^{3+}{ }^{5} \mathrm{I}_{7}$ level (with a greater rate than that of radiative relaxation $[13,27,28]$ ), the detected signal as a measure of lifetime is quite low to serve for accurate measuring of $\tau_{2}$ using short HDF sections. Thus, to get a well-detectable photodetector signal, one usually deals with a relatively long HDF piece and a high pump level, but these measures eventually result in irrelevant ambiguities in the experimental data interpretation.

Fig. 4 shows the SE decays simulated using formula (3) for the two different values of the initial inversion population $\left(n_{20}=0.5\right.$ and 1.0) and for the broad range of $O D$-values $(0.01$ to 2.0).

Note that the case when $n_{20}=1$ corresponds to a fully saturated $\mathrm{Ho}^{3+}$ laser level, assessible at very high pump powers; in turn, when $n_{20}=0.5$ the fiber gain compensates the fiber absorption $\left(\alpha_{0} n_{1}=g_{0} n_{2}\right)$, but this condition is hardly controllable. 

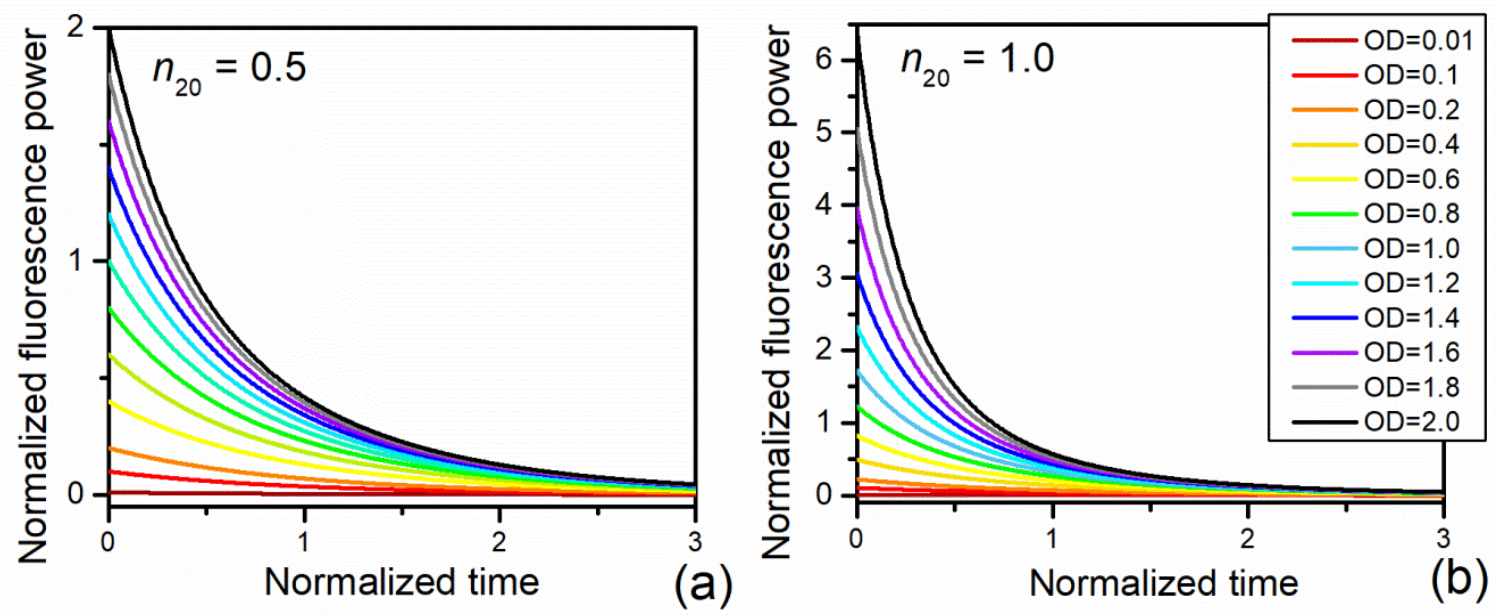

Figure 4. Fluorescence decays normalized to $K_{s e}$, simulated at (a) $n_{20}=0.5$ and (b) $n_{20}=1$. The $O D$-values used for generating both plots are shown in the inset to (b). In both plots, the time scale is normalized to the real lifetime $\tau_{2}$.

As seen from Fig. 4, the fluorescence power dramatically increases with increasing both $O D$ and $n_{20}$. But, by making single exponential fits for all shown curves, one can find that the values of SE decay are $O D$-dependent, see Fig. 5(a). At very low $O D$, it is equal to the real fluorescence lifetime while with increasing $O D$ it drops down to the values dependent on the initial population inversion $n_{20}$ : the higher $n_{20}$ is, the stronger lifetime decreases. When the laser level is fully saturated $\left(n_{20}=1\right)$, the estimated SE lifetime decreases by approximately three times at increasing of $O D$ from 0.01 to 2 (see the orange curve in Fig. 5(a)). At lower $n_{20}$, it decreases in a lesser degree (see the blue curve simulated for $n_{20}=0.5$ ). At the same time, the residual sum $\mathrm{R}^{2}$ of the fitting is always relatively high, close to 1 (refer to Fig. 5(b)). Note that the latter simulations were made without consideration of photodetector noise that vastly decreases $\mathrm{R}^{2}$.
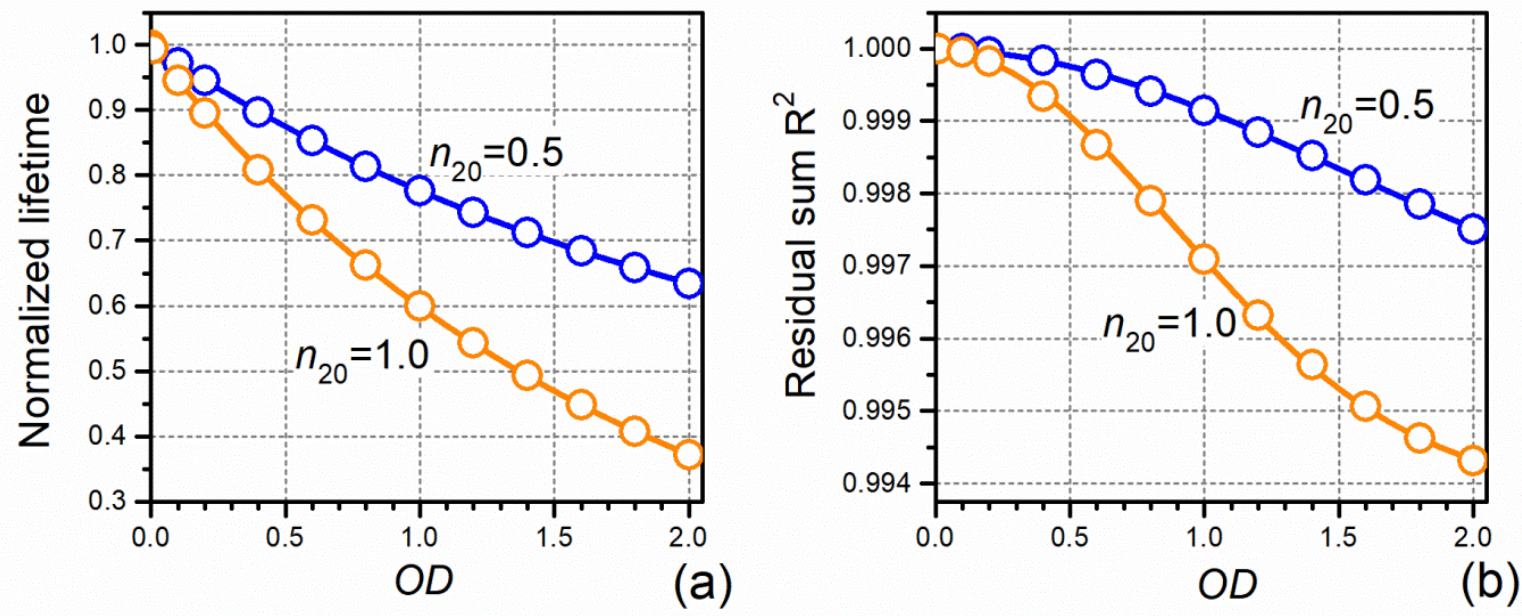

Figure 5. (a) Normalized lifetime as a function of $O D$-value. (b) Residual sum of the exponential fit, applied to the fluorescence decay functions shown in Fig. 4(a). In both plots, circles stand for the simulated points and lines show the fits by (a) exponential decay and (b) Gaussian functions.

Fig. 6 demonstrates the fluorescence decays shown in Fig. 4(b) (for $n_{20}=1$ ) but with the vertical axis scaled logarithmically. In the figure, are also shown three groups of symbols, connected by lines. Each of these groups adhere to the points, from which the dependencies are 
to be fit for getting fluorescence lifetime $\tau_{2}$ with the error labelling near each set. As seen, the smaller the error, the longer is the initial section to be cut. Importantly, the fluorescence signal obtained for a higher $O D$-value should be cut at a lower value than the uncut fluorescence signal obtained for a lower $O D$-value. For instance, the whole fluorescence decay measured at $O D=$ 0.1 permits one to get $\tau_{2}$ with $5 \%$ error, whereas that measured at $O D=2$ should be cut at the level two times lower for the same error. In other words, the use of the long fiber pieces does not allow one to get fluorescence time with acceptable error by applying a single exponential decay fit. An example of getting fluorescence time using formula (3) will be discussed below.

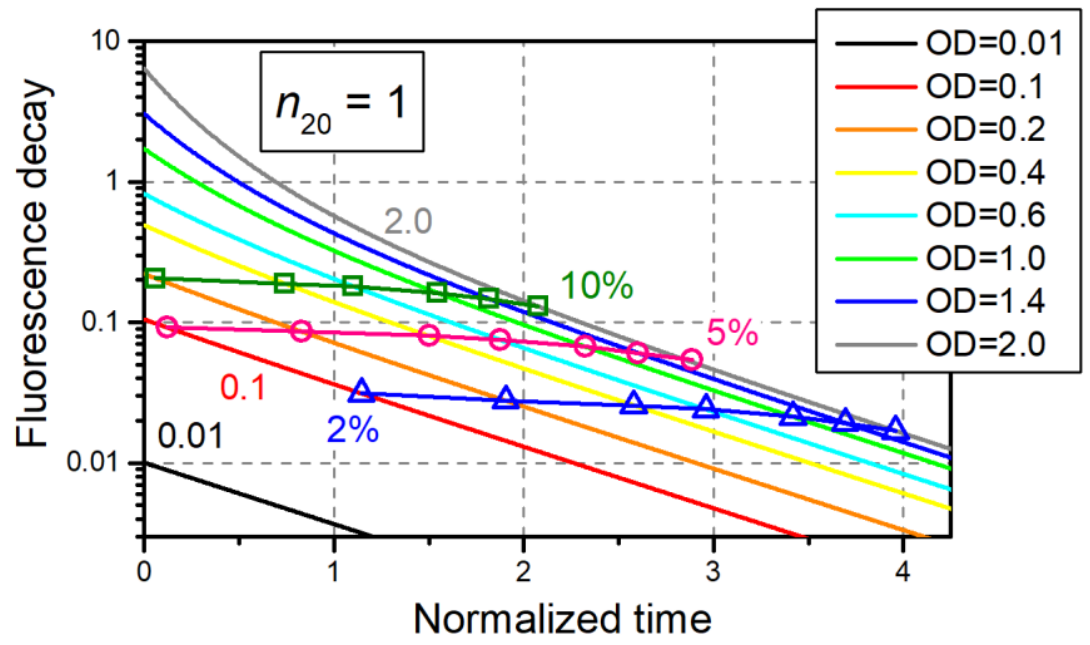

Figure 6. Fluorescence decay functions, simulated for different $O D$-values (indicated in the inset) at $n_{20}=1$; some of the dependences are labeled with $O D$-values. Three sets of symbols (triangles, circles, and rectangles) and connecting lines indicate the points, above which the dependencies should be cut for making it possible determination of the fluorescence time with errors of $2 \%, 5 \%$, or $10 \%$.

\subsection{Theoretical background: measurement of decay constant of ${ }^{5} I_{6}$ level}

Let us explore the case of a CW-pumped HDF section where the pump level $\left({ }^{5} \mathrm{I}_{6}\right)$ depletes, after switching off the pump light (at $t=0$ ), via phonon decay to the laser level $\left({ }^{5} \mathrm{I}_{7}\right)$. We consider here that $O D$ of $\mathrm{HDF}$ is not too large and that lifetimes of all upper (than ${ }^{5} \mathrm{I}_{6}$ ) levels are very short (hence their populations are zero). In this case, the fluorescence decay is described by the simplified three-level scheme that only involves the three lowest levels in Fig. 2. Their populations adhere the following three balance equations:

$$
\begin{gathered}
\frac{d N_{3}}{d t}=\frac{\sigma_{13} I_{p}}{h v_{p}} N_{1}-\frac{N_{3}}{\tau_{3}} \\
\frac{d N_{2}}{d t}=\frac{N_{3}}{\tau_{3}}-\frac{N_{2}}{\tau_{2}} \\
N_{1}+N_{2}+N_{3}=N_{0}
\end{gathered}
$$

where $N_{0}$ is the concentration of $\mathrm{Ho}^{3+}$ ions in the fiber core and $N_{i}(i=1$ to 3 ) are the populations of these levels, $h v_{p}$ is the pump photon energy, $\sigma_{13}$ is the absorption cross-section of $\mathrm{Ho}^{3+}$ at the pump wavelength $(1.134 \mu \mathrm{m})$, and $\tau_{2}$ and $\tau_{3}$ stand for the decays of levels 2 and 3 ( $\tau_{2}$ is the fluorescence lifetime and $\tau_{3}$ is the nonradiative decay on phonons).

As the HDF section under study is CW-pumped, the derivatives in Eqs. (5a), (5b) are to be zeroed, which results in the following expressions for the normalized $\left(n_{i}=N_{i} / N_{0}\right)$ populations: 


$$
\begin{gathered}
n_{1}=\frac{1}{1+(1+\xi) s_{p}} \\
n_{2}=\frac{s_{p}}{1+(1+\xi) s_{p}} \\
n_{3}=\frac{\xi s_{p}}{1+(1+\xi) s_{p}}=\xi n_{2}
\end{gathered}
$$

where $\xi=\tau_{3} / \tau_{2}=n_{3} / n_{2} \ll 1, s_{p}=P_{p} / P_{p}^{\text {sat }}$ is the pump saturation parameter, $P_{p}$ is pump power, $P_{p}^{\text {sat }}=\left(h v_{p}\right) A_{\text {eff }} f\left(\sigma_{13} \tau_{2}\right)$ is the saturation power at the pump wavelength, and $A_{\text {eff }}$ is the effective modal area at the pump wavelength.

The obtained normalized populations should be used as the initial ones just before decaying after switching pump off. The population of the third (pump) level is described by Eq. (5a) where $I_{p}=0$; its solution is the exponential decay function: $n_{3}(t)=n_{30} \exp \left(-t / \tau_{3}\right)$. Accordingly, the population of the second (laser) level is also described by the exponential decay function, but the decay process is superimposed with this level's populating during the time interval (of the order of a few $\tau_{3}$ ) owing to continued decay of the third level:

$$
\frac{d n_{2}(t)}{d t}=\frac{n_{30} \exp \left(-\frac{t}{\tau_{3}}\right)-n_{2}(t)}{\tau_{2}}
$$

The approximate solution of the last equation is:

$$
n_{2}(t)=n_{20}\left[1+\xi\left(1-\exp \left(-\frac{t}{\tau_{3}}\right)\right)\right] \exp \left(-\frac{t}{\tau_{2}}\right)
$$

The curves, simulated using formula (7b) for different ratios of $\xi=\tau_{3} / \tau_{2}$, are shown in Fig. 7. As seen, if $\tau_{3}$ is negligible $\left(\xi=10^{-3}\right.$ ), the signal of $\sim 2-\mu \mathrm{m}$ fluorescence (proportional to $n_{2}$ ) decays with the classical single exponential law. With increasing $\tau_{3}$ the decay function shifts in Fig. 7 upper and to the right side (by approximately $\xi$-value), with smooth transition area in the time domain from 0 to $\sim 3 \xi$. This effect is explained by the fact that after switching pump off the level ${ }^{5} \mathrm{I}_{7}$ is still populating, but with decreasing rate, via nonradiative decay from the level ${ }^{5} \mathrm{I}_{6}$ until the latter becomes empty.

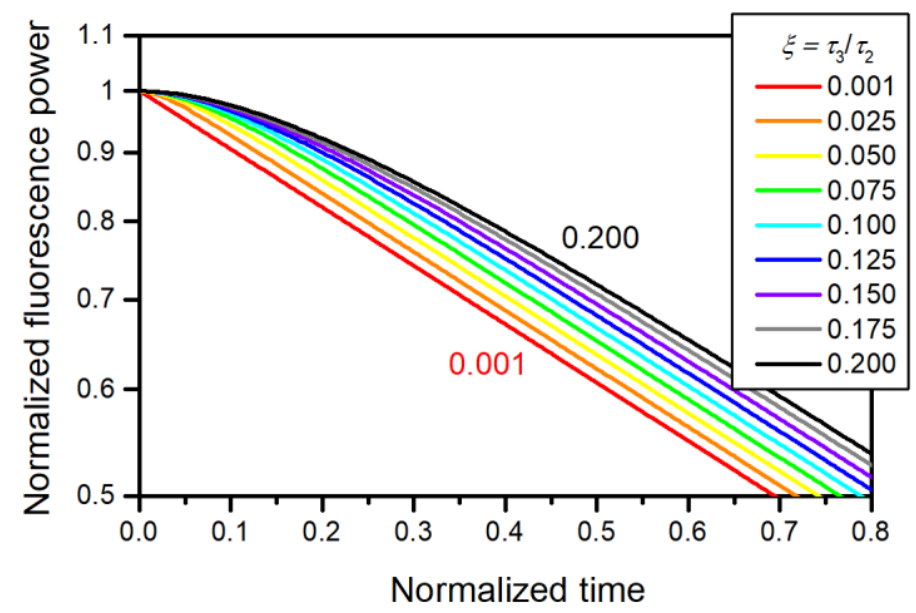

Figure 7. Simulated $\sim 2-\mu \mathrm{m}$ fluorescence decay after switching off the pump light. Time scale is normalized to the lifetime $\tau_{2}$. The vertical axis is logarithmic. The curves simulated for $\xi=0.001$ and 0.200 are labeled in the inset. 


\subsection{Measurement of decay constant of ${ }^{5} I_{6}$ level and fluorescence lifetime in HDF}

First, let us explore the application of CDF for measuring the fluorescence lifetime $\tau_{2}$ when the fiber under study is relatively long so that the condition $O D \ll<1$ is not satisfied. For measuring $\tau_{2}$, we used a HDF piece with $O D=1$ that permitted us to have experimentally the fluorescence signal of a high magnitude, for more reliable measurements. The experimental fluorescence decay is shown in both panels of Fig. 8 by gray circles. Here, the primary section of the experimental curve, from zero to $100 \mu \mathrm{s}$, was cut out as it presents the smooth transition area, discussed above (refer to Fig. 7). For better understanding the matter, we show the results of fitting using two functions: the single exponential decay (see the blue curve in plot (a)) and the CDF built using Eq. (3) (see the yellow curve in plot (b)). The results of the measurements are compared in Table 1.
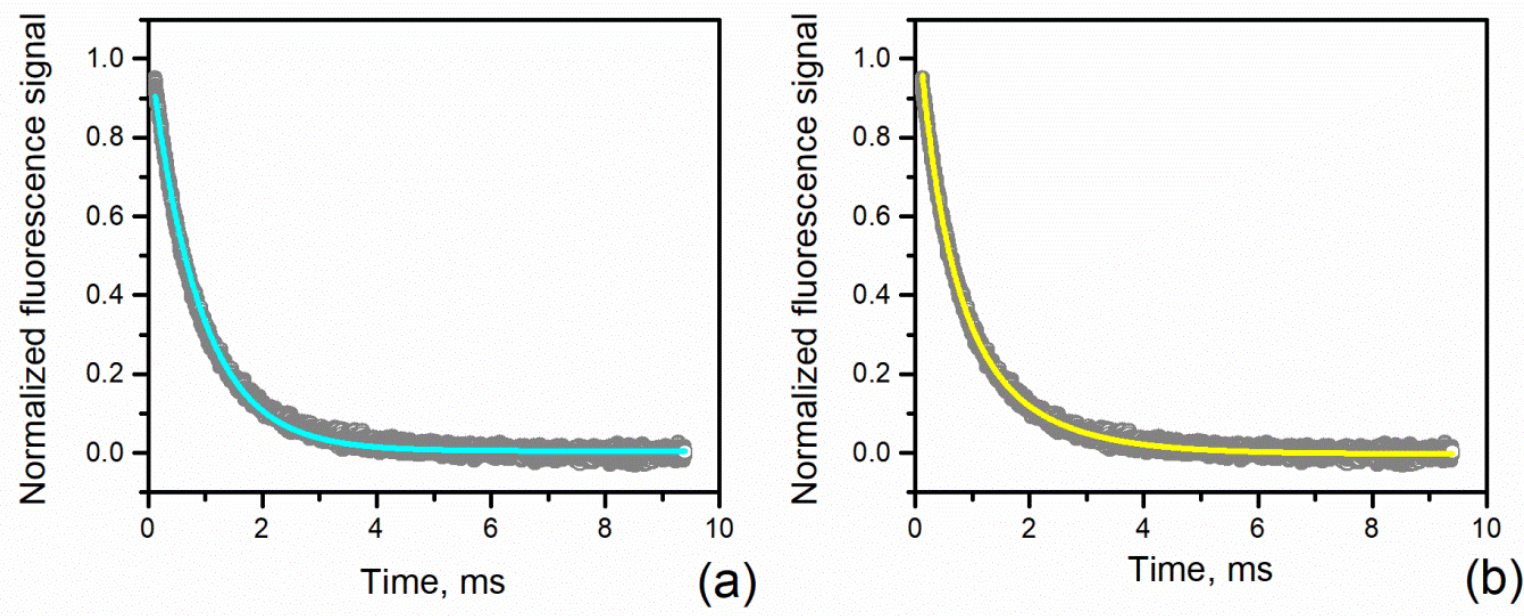

Figure 8. Fluorescence decay measured using a HDF section with OD = 1 (gray symbols) and its fits (solid lines), approximated by (a) exponential decay function and (b) CDF.

Table 1

Fluorescence decay parameters

\begin{tabular}{|c|c|c|}
\hline & Exponential decay & $\mathrm{CDF}$ \\
\hline$\tau_{2}, \mathrm{~ms}$ & 0.86 & 1.4 \\
\hline $\mathrm{R}^{2}$ & 0.9954 & 0.9976 \\
\hline$O D$ & - & 0.996 \\
\hline$n_{20}$ & - & 0.98 \\
\hline
\end{tabular}

From Table 1, one can see that the fluorescence lifetime measured using the exponential decay function is by $\sim 40 \%$ less than that found using CDF, although the residual sums $\mathrm{R}^{2}$ of these two fits are closed to 1 (compare these results with the data presented in Fig. 5). It is also seen that $\mathrm{CDF}$, besides the fluorescence lifetime, permits one to obtain some other parameters, useful for modeling HDFL, such as the $O D$-value of the fiber piece under test and, also, the 
initial normalized population of the laser level. Note that the found fluorescence lifetime, $\tau_{2}=1.4$ $\mathrm{ms}$, is slightly above its highest experimental value for HDFs, measured in lateral geometry of fluorescence capture; see ref. [30]

Second, let us assess the fine details, expected to occur (as per the modeling results presented in Fig. 7) within the primary segment of fluorescence decay that was cut out in the plots shown in Fig. 8. As mentioned above, this segment contains the information about population of the third $\mathrm{Ho}^{3+}$ level $n_{30}$ and its effective depletion time $\tau_{3}$. For seeking for estimates for these two quantities, we zoomed a relatively short part of the whole experimental fluorescence decay, obtained at $O D=1$; see Fig. 9. As seen, there exist the two components here: $(i)$ the fast transitional area (marked by the ellipse) and (ii) the relatively slow exponential decay (linear in the semi-logarithmic plot used). (Note that since depletion of the third level does not populate the ground level, the effect of reabsorption does not contribute to the data plotted.)

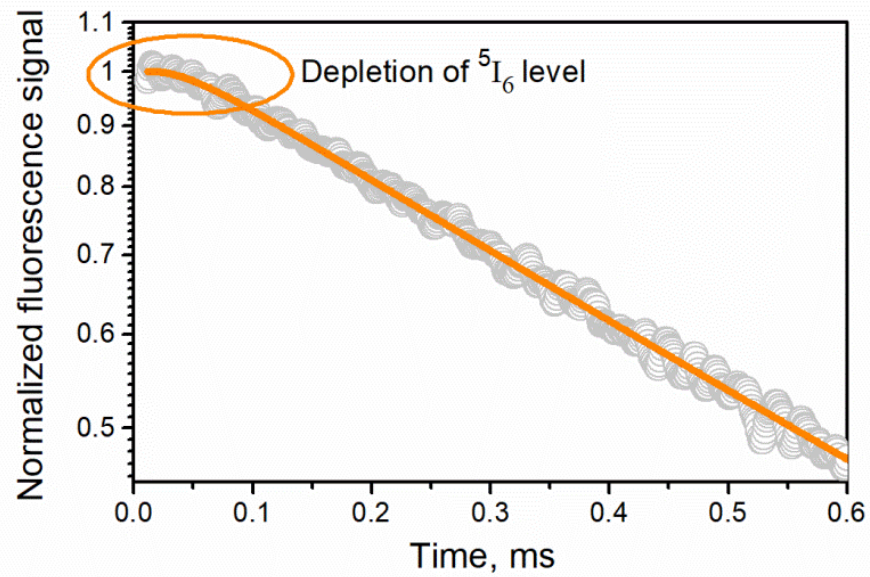

Figure 9. The primary segment of $\sim 2-\mu \mathrm{m}$ fluorescence decay.

Applying Eq. 7(b) to fit the fluorescence decay within its short segment shown by gray circles in Fig. 9, we obtained the following estimates: $\tau_{3} \approx 25 \mu$ s and $n_{30} \approx 0.02$. Certainly, the proposed method for measuring the fast contribution in overall decay of $\sim 2-\mu \mathrm{m}$ fluorescence of the HDF is not straightforward but it is capable in bringing in more clarity about the processes involved, which may be useful for further, more detailed, modeling of HDFLs.

\section{Conclusions}

In this work, we assessed the spectroscopic properties of home-made silica-based holmiumdoped fiber (HDF), co-doped with aluminum and germanium, for laser applications. The HDF's broadband ground-state absorption spectrum, the excited-state absorption spectra under pumping at $1.12 \mu \mathrm{m}$, and the fluorescence spectrum under laser action were measured and examined. We also developed a reliable method for measuring $\mathrm{Ho}^{3+}$ fluorescence lifetime at $\sim 2 \mu \mathrm{m}$, which accounts for the decay of fluorescence itself from the "laser" $\left({ }^{5} \mathrm{I}_{7}\right)$ level and, also, the nonradiative (multi-phonon) decay of excitation of the "pump" $\left({ }^{5} \mathrm{I}_{6}\right)$ level. The analytical formulae, potentially useful for precise modeling of HDF-based lasers, were derived for unambiguous fitting the experimental fluorescence decay. Particularly, we showed that the use of single exponential decay function for fitting fluorescence decay in the presence of notable optical depth $(O D)$ of a fiber under test inevitably results in errors. Say, even at rather small $O D(O D=$ 
0.1 ) this leads to errors in lifetime measurements of $\sim 5 \%$ but at increasing $O D$ up to 1 the errors reach $\sim 40 \%$ and at $O D=2$ the errors can become as large as $\sim 3$ times. Furthermore, application of the developed formula allowed us to get reliable fluorescence lifetime of $1.4 \mu$ s for our HDF. We also demonstrated that the characteristic time of non-radiative (multi-phonon) decay of the pump $\left({ }^{5} \mathrm{I}_{6}\right) \mathrm{Ho}^{3+}$ level is $\sim 25 \mu \mathrm{s}$. The results reported herein can be useful for numerical optimization of HDF-based $\sim 2-\mu \mathrm{m}$ lasers while the formulae derived for fitting fluorescence decay applicable for active fibers of other types. The formulas derived in the presented work are useful also for fitting fluorescence decay of fibers doped with other rare-earth ions.

\section{References}

[1] Mingareev, I., Weirauch, F., Olowinsky, A., Shah, L., Kadwani, P., Richardson, M. 2012. Welding of polymers using a $2 \mu \mathrm{m}$ thulium fiber laser. Optics \& Laser Technology 44: 2095-2099.

[2] Sincore, A., Bradford, J. D., Cook, J., Shah, L., and Richardson, M. C. 2018. High average power thulium-doped silica fiber lasers: Review of systems and concepts, IEEE Journal of Selected Topics in Quantum Electronics 24(3):0901808.

[3] Mingareev, I., Gehlich N., Bonhoff T., Abdulfattah, A., Sincore, A. M., Kadwani, P., Shah, L., Richardson, M. 2016. Principles and applications of trans-wafer processing using a 2- $\mu \mathrm{m}$ thulium fiber laser. The International Journal of Advanced Manufacturing Technology 84:2567-2578.

[4] Petros, M., Singh, U. N., Yu, J., Kavaya, M. J., and Koch, G. 2014. 2-micron Coherent Doppler Lidar Instrument Advancements for Tropospheric Wind Measurement. Proceedings of SPIE 9246:92460A.

[5] McComb, T. S., Sims, R. A., Willis, C. C. C., Kadwani, P., Shah, L., and Richardson M. 2010. Atmospheric transmission testing using a portable, tunable, high power thulium fiber laser system. Proceeding of Conference on Lasers Electro-Optics JThJ5.

[6] Dong, L. and Samson, B. 2017. Fiber Lasers: Basics, Technology, and Applications. Boca Raton: CRC Press.

[7] Hanna, D. C., McCarthy, M. J., Perry, I. R., and Suni, P. J. 1989. Efficient high-power continuouswave operation of monomode Tm-doped fiber laser at $2 \mu \mathrm{m}$ pumped by Nd-YAG laser at 1.064 $\mu \mathrm{m}$. Electronics Letters 25(10):1365-1366.

[8] Barnes, W. L. and Townsend, J. E. 1990. Highly tunable and efficient diode pumped operation of $\mathrm{Tm}^{3+}$ doped fiber lasers. Electronics Letters 26(11): 746-747.

[9] Hanna, D. C., Percival, R. M., Smart, R. G., Townsend, J. E., and Tropper, A. C. 1989. Continuous-wave oscillation of holmium-doped silica fiber laser. Electronics Letters 25(9): 593594.

[10] Allain, J. Y., Monerie, M., and Poignant, H. 1991. High-efficiency CW thulium-sensitized holmium-doped fluoride fiber laser operating at $2.04 \mu \mathrm{m}$. Electronics Letters 27(17):1513-1515.

[11] Holmen, L. G., Shardlow, P. C., Barua, P., Sahu, J. K., Simakov, N., Hemming, A., and Clarkson, W. A. 2019. Tunable holmium-doped fiber laser with multiwatt operation from $2025 \mathrm{~nm}$ to 2200 nm. Optics Letters 44(17): 4131-4134.

[12] Wu, K. S., Ottaway, D., Munch, J., Lancaster, D. G., Bennetts, S., and Jackson S. D. 2009. Gainswitched holmium-doped fibre laser. Optics Express 17(23): 20872-20877.

[13] Baker, C. C., Friebele, E. J., Burdett, A. A., Rhonehouse, D. L., Fontana, J., Kim, W., Bowman, S. R., Shaw, L. B., Sanghera, J., Zhang, J., Pattnaik, R., Dubinskii, M., Ballato, J., Kucera, C., Vargas, A., Hemming, A., Simakov, N., and Haub, J. 2017. Nanoparticle doping for high power fiber lasers at eye-safer wavelengths. Optics Express 25(12):13903-13915.

[14] Hemming, A., Simakov, N., Haub, J., and Carter A. 2014. A review of recent progress in holmiumdoped silica fibre sources. Optical Fiber Technology 20(6): 621-630. 
[15] Kurkov, A. S., Dvoyrin, V. V., and Marakulin, A. V. 2010. All-fiber $10 \mathrm{~W}$ holmium lasers pumped at $\lambda=1.15 \mu \mathrm{m}$. Optics Letters 35(4):490-492.

[16] Kamynin, V. A., Kablukov, S. I., Raspopin, K. S., Antipov, S. O., Kurkov, A. S., Medvedkov, O. I., and Marakulin, A. V. 2012. All-fiber Ho-doped laser tunable in the range of 2.045-2.1 $\mu \mathrm{m}$, Laser Physics Letters 9:893.

[17] Kir'yanov, A. V., Barmenkov, Y. O., and Villegas Garcia, I. 2017. $2.05 \mu \mathrm{m}$ holmium-doped allfiber laser diode-pumped at 1.125 $\mu \mathrm{m}$. Laser Physics 27:085101.

[18] Kir'yanov, A. V., Barmenkov, Y. O., Villegas-Garcia, I. L. Cruz, J. L., and Andres, M. V. 2018. Highly Efficient Holmium-Doped All-Fiber 2.07- $\mu$ m Laser Pumped by Ytterbium-Doped Fiber Laser at $\sim 1.13 \mu \mathrm{m}$. IEEE Journal of Selected Topics in Quantum Electronics 24(5):0903108.

[19] Xiao, H., Zhang, H., Xu, J., Leng, J., and Zhou P. 2017. $120 \mathrm{~W}$ monolithic Yb-doped fiber oscillator at $1150 \mathrm{~nm}$. Journal of the Optical Society of America B 34(3):A63-A69.

[20] Golant, K. M. 2007. Surface plasma chemical vapor deposition: 20 years of application in glass synthesis for lightguides (a review). Proceeding of XXI International Congress on Glass L13.

[21] Simakov, N., Li, Z., Jung, Y., Daniel, J. M. O., Barua, P., Shardlow, P. C., Liang, S., Sahu, J. K., Hemming, A., Clarkson, W. A., Alam, S.-U., and Richardson, D. J. 2016. High gain holmiumdoped fibre amplifiers. Optics Express 24(23):13946-13956.

[22] Kamrádek, M., Kašík, I., Aubrecht, J., Mrázek, J., Podrazký, O., Cajzl, J., Vařák, P., Kubeček, V., Peterka, P., Honzátko, P. 2019. Nanoparticle and Solution Doping for Efficient Holmium Fiber Lasers [invited paper]. Photonics Journal 11(5): 7103610.

[23] Friebele, E. J., Askins, C. G., Peele, J. R., Wright, B. M., Condon, N. J., O’Connor, S., Brown, C. G., and Bowman, S. R. 2014. Ho-Doped Fiber for High Energy Laser Applications. Proceedings of SPIE 8961:896120.

[24] Simakov, N., Hemming, A., Clarkson, W. A., Haub, J., and Carter, A. 2013. A cladding-pumped, tunable holmium doped fiber laser. Optics Express 21(23):28415-28422.

[25] Wang, X., Hu, L., Xu, W., Wang, S., Zhang, L., Yu, C., and Chen, D. 2015. Spectroscopic properties of $\mathrm{Ho}^{3+}$ and $\mathrm{Al}^{3+}$ co-doped silica glass for 2- $\mu \mathrm{m}$ laser materials. Journal of Luminescence 166:276-281.

[26] Ryabochkina, P. A., Chabushkin, A. N., Kosolapov, A. F., and Kurkov, A. S. 2015. Absorption and luminescence characteristics ${ }^{5} \mathrm{I}_{7} \rightarrow{ }^{5} \mathrm{I}_{8}$ transitions of the holmium ion in $\mathrm{Ho}^{3+}$-doped aluminosilicate preforms and fibres. Quantum Electronics 45(2):102-104.

[27] Layne, C. B., Lowdermilk, W. H., and Weber, M. J. 1977. Multiphonon relaxation of rare-earth ions in oxide glasses. Physical Review B 16(1):10-20.

[28] Burshtein, Z. 2010. Radiative, nonradiative, and mixed-decay transitions of rare-earth ions in dielectric media. Optical Engineering 49(9):091005.

[29] Bowman, S. R., O’Connor, S., Condon, N. J., Friebele, E. J., Kim, W., Shaw, B., and Quimby R. S. 2013. Non-radiative decay of holmium-doped laser materials. Proceedings of SPIE 8638:863803.

[30] Wang, X., Xu, W., Wang, S., Yu, C., Chen, D., and Hu, L. 2016. Journal of Alloys and Compounds 657:478-482.

[31] Kamrádek, M., Aubrecht, J. Peterka, P., Podrazký, O., Honzátko, P., Cajzl, J., Mrázek, J., Kubeček, V., and Kašík I. 2019. Spectroscopic characterization of holmium-doped optical fibers for fiber lasers. Proceedings of SPIE 11029:1102908.

[32] Kamrádek, M., Kašík, I., Aubrecht, J., Mrázek, J., Podrazký, O., Cajzl, J., Varák, P., Kubeček, V., Peterka, P., and Honzátko P. 2020. Holmium-doped optical fibers for efficient fiber lasers. Proceedings of SPIE 11355:113550C.

[33] Watekar, P. R., Ju, S., Han, W.-T. 2008. Optical properties of Ho-doped alumino-germano-silica glass optical fiber. Journal of Non-Crystalline Solids 354:1453-1459.

[34] Kolpakov, S. A., Barmenkov, Y. O., Guzman-Chavez, A. D., Kir'yanov, A. V., Cruz, J. L., Díez, A., and Andrés, M. V. 2011. Distributed Model for Actively Q-Switched Erbium-Doped Fiber Lasers. IEEE Journal of Quantum Electronics 47(7):928-934. 


\section{Acknowledgement}

This work was supported in part by the Ministerio de Ciencia e Innovación of Spain and the Fondo Europeo de Desarrollo Regional (Ref.: PID2019-104276RB-I00). 